\title{
Adaptaciones al ejercicio físico en el perfil lipídico y la salud cardiovascular de obesos mórbidos
}

\author{
Pedro Delgado-Floody', Felipe Caamaño-Navarrete², Daniel Jerez-Mayorga³, Cristian Martínez-Salazar', \\ Felipe García-Pinillos' ${ }^{1}$ y Pedro Latorre-Román ${ }^{4}$ \\ ${ }^{1}$ Departamento de Educación Física, Universidad de La Frontera, Temuco; ${ }^{2}$ Carrera de Pedagogía en Educación Física, Facultad de Educación, \\ Universidad Católica de Temuco, Temuco; ${ }^{3}$ Facultad de Ciencias de la Rehabilitacion, Universidad Andres Bello, Santiago, Chile; ${ }^{4}$ Departamento \\ de Didáctica de la Expresión Musical, Plástica y Corporal, Universidad de Jaén, Jaén, España
}

\begin{abstract}
Resumen
Objetivo: Evaluar los efectos de un programa de ejercicio físico sobre el perfil lipídico, el estado ponderal y la salud cardiovascular de obesos candidatos a cirugía bariátrica. Método: Veintidós obesos mórbidos participaron de un programa de ejercicio físico de 6 meses de duración. Dieciséis formaron el grupo adherente (asistencia $\geq 80 \%$; edad: $37.81 \pm 11.90$ años) y seis el grupo no adherente (asistencia < 80\%; edad: $45.83 \pm 14.23$ años). Antes y 72 horas después de la última sesión se evaluaron el peso, el índice de masa corporal (IMC), el contorno de la cintura (CC), la presión arterial, la glucemia basal, la capacidad cardiorrespiratoria, el colesterol y los triglicéridos. Resultados: El peso, el IMC y el CC presentaron cambios significativos $(p<0.05)$ en ambos grupos, siendo mejores los resultados del grupo adherente. En el grupo adherente, además, aumentó la capacidad cardiorrespiratoria $(p=0.001)$ y disminuyeron significativamente la presión diastólica $(p=$ $0.011)$, la glucemia basal $(p=0.021)$ y los triglicéridos $(p<0.001)$. El grupo no adherente no presentó cambios significativos en estas variables $(p \geq 0.05)$. Conclusión: La intervención fue factible de realizar sin efectos negativos para los participantes, y se puede recomendar para mejorar la salud cardiovascular de estos pacientes.
\end{abstract}

PALABRAS CLAVE: Obesidad. Obesidad mórbida. Ejercicio físico.

\begin{abstract}
Objective: To assess the effects of a physical exercise program on the lipid profile, weight status and the cardiovascular health of obese candidates for bariatric surgery. Method: 22 morbidly obese patients participated in a 6-month physical exercise program. 16 formed the adherent group (attendance $\geq 80 \%$; age: $37.81 \pm 11.90$ years) and six the non-adherent group (attendance < 80\%; age: $45.83 \pm 14.23$ years). Before and $72 \mathrm{~h}$ after the last session were evaluated: weight, body mass index (BMI), contour waist, blood pressure, fasting, cardio respiratory fitness, cholesterol and triglycerides. Results: Weight, $B M I$ and WC showed significant changes $(p<0.05)$ in both groups, with the results of the adherent group being better. In the adherent group, cardiorespiratory capacity $(p=0.001)$ was also increased, while diastolic pressure $(p=0.011)$, basal glycemia $(p=0.021)$ and triglycerides decreased significantly $(p<0.001)$. The non-adherent group did not present significant changes in these variables $(p \geq 0.05)$. Conclusions: The intervention was feasible to perform without negative effects for the participants, and it is possible to recommend improving the cardiovascular health of these of patients.
\end{abstract}

KEY WORDS: Obesity. Morbid obesity. Physical exercise.

\author{
Correspondencia: \\ Pedro Delgado-Floody \\ Calle Uruguay n 1980 \\ C.P 4780000 , Temuco, Araucanía, Chile \\ E-mail: pedro.delgado@ufrontera.cl
}

Fecha de recepción en versión modificada: 29-11-2016

Fecha de aceptación: 04-01-2017

DOI://dx.doi.org/10.24875/GMM.17002894
Gac Med Mex. 2017;153:781-786

Contents available at PubMed www.gacetamedicademexico.com 


\section{Introducción}

La obesidad promueve una serie de procesos celulares que atenúan la señalización de la leptina, desarrollando la "resistencia a la leptina celular» y amplificando el grado de ganancia de peso $^{1}$, y desempeñando un papel en la regulación de los depósitos de grasa corporal y los valores de glucosa en la sangre ${ }^{2}$. Se considera como una de las enfermedades no transmisibles más importantes y prevalentes en la actualidad ${ }^{3}$, se asocia con los factores de riesgo de enfermedad cardiovascular ${ }^{4,5}$ y con algunos tipos de cáncer $^{6}$, afecta a la respuesta inmunitaria ${ }^{7}$, conduce a un mayor estado de morbilidad y mortali$\mathrm{dad}^{8}$, y produce una disminución considerable de la expectativa de vida ${ }^{9}$.

La obesidad mórbida es una enfermedad crónica con graves consecuencias en la salud por la comorbilidad asociada, y constituye un factor de riesgo desencadenante del síndrome metabólico y de enfermedad cardiovascular ${ }^{10}$. En Chile, la obesidad se ha duplicado en las últimas décadas, y la obesidad mórbida en específico aumentó de 148,000 personas en el año 2003 a 300,000 en el año 201011, generando elevados costos para la salud pública.

Para el tratamiento de la obesidad mórbida, la cirugía bariátrica es una técnica con la que se consigue una gran mejoría en los parámetros antropométricos, en los factores de riesgo cardiovascular y en la calidad de vida de los pacientes ${ }^{12}$; sin embargo, es importante considerar que un mayor peso preoperatorio podría aumentar la morbimortalidad ${ }^{13}$, generando la necesidad de tratamientos preoperatorios que mejoren esta condición y adapten a los pacientes a las etapas posteriores. Existe evidencia de que el ejercicio físico intervalado y la asesoría nutricional mejoran estas condiciones, pero existe escasa información que permita validar programas para el tratamiento preoperatorio, generando la necesidad de implementar intervenciones estructuradas.

El objetivo de esta investigación fue evaluar los efectos de un programa de ejercicio físico sobre el perfil lipídico, el estado ponderal y la salud cardiovascular de obesos candidatos a cirugía bariátrica.

\section{Método}

Veintidós obesos/obesos mórbidos de entre 18 y 60 años participaron de forma voluntaria y se les aplicó un programa de ejercicio físico de 6 meses de duración con tres estímulos por semana (1 hora por sesión). Cada paciente fue control de sí mismo antes y después de la intervención. Dieciséis sujetos formaron el grupo adherente (edad: $37.81 \pm 11.90$ años) y seis el grupo no adherente (edad: $45.83 \pm 14.23$ años).

En los criterios de inclusión se estableció que los sujetos tuvieran entre 18 y 60 años de edad, un índice de masa corporal (IMC) $\geq 40 \mathrm{~kg} / \mathrm{m}^{2}$ o un IMC $\geq 35 \mathrm{~kg} / \mathrm{m}^{2}$ y comorbilidades. (diabetes mellitus tipo II, hipertensión arterial, dislipidemia, resistencia a la insulina), habilitación médica para la práctica de ejercicio físico y no participar de programas previos de actividad física. Se solicitó una asistencia $\geq 80 \%$ del total de las sesiones programas.

Los criterios de exclusión de la investigación fueron presentar limitaciones físicas para realizar ejercicio, presencia de patologías respiratorias, enfermedades cardiacas crónicas con alguna agudización, lesiones o patologías en el sistema osteomioarticular que impidan la realización de ejercicios físicos, o enfermedades crónicas no transmisibles descompensadas 0 no controladas.

De los 22 participantes que iniciaron la intervención, 16 cumplieron con los requerimientos estipulados y formaron el grupo adherente. Los pacientes que tuvieron participación $<80 \%$ del total de las sesiones del programa pasaron a formar el grupo no adherente $(n=6)$; estos sujetos participaron del programa durante todos los meses, pero de forma irregular, con un $30 \%$ de asistencia promedio (22 sesiones).

En la investigación se han respetado los acuerdos de la Declaración de Helsinki (2013) y todo paciente firmó un consentimiento informado antes del estudio.

Los pacientes fueron citados durante un día para realizar las evaluaciones preintervención. Entre las 8:00 y las 10:00 de la mañana se recolectaron las variables del perfil lipídico en ayuno y presión arterial; 8 horas después se midieron las variables del estado ponderal y se aplicó el test de la marcha de 6 minutos (6MWT). Las mediciones finales se realizaron 72 horas después de ejecutada la última sesión de ejercicios con el mismo orden utilizado en el pretest. Se instruyó a todos los sujetos no modificar sus hábitos de alimentación ni su actividad física durante la intervención.

El peso se determinó con una balanza calibrada de palanca de adulto mecánica $(220 \mathrm{~kg})$ con tallímetro, graduada, de marca SECA ${ }^{\circledR}$. La balanza se calibró cada vez que se evaluó a un individuo según el manual del fabricante. EI IMC se determinó para estimar 
el grado de obesidad. El contorno de la cintura (CC) se midió con una cinta métrica retráctil de adulto graduada en centímetros, marca SECA ${ }^{\circledR}$.

La evaluación de los parámetros bioquímicos se realizó mediante muestras sanguíneas recolectadas en tubos sin anticoagulante. Las muestras se centrifugaron a 2500 r.p.m. por 10 minutos, para obtener el suero a utilizar. Las concentraciones séricas de glucosa, colesterol total, colesterol ligado a lipoproteínas de alta densidad (c-HDL), colesterol ligado a lipoproteínas de baja densidad (c-LDL) y triglicéridos se determinaron por métodos colorimétricos estándar en un autoanalizador HumaStar80. La concentración de c-LDL se obtuvo mediante la fórmula de Friedwald, cuando la concentración de triglicéridos fue $<400 \mathrm{mg} /$ dl. Las muestras fueron tomadas y procesadas por tecnólogos médicos en los centros de salud de cada paciente con un ayuno $\geq 10$ horas.

La presión arterial fue medida con el protocolo de la guía clínica de hipertensión primaria o esencial en personas de 15 años y más ${ }^{14}$. Para evaluar la capacidad cardiorrespiratoria se utilizó el 6MWT debido a que es una herramienta cada vez más utilizada en la práctica clínica que evalúa la capacidad para realizar ejercicio físico de un sujeto, midiendo la distancia máxima que puede caminar durante 6 minutos. El protocolo del 6MWT se realizó en un espacio cerrado, de superficie plana, de 30 metros de longitud, en cuyos extremos se instalaron dos conos reflectantes para señalar el límite de recorrido y permitir que los sujetos obesos mórbidos giraran alrededor de ellos. En el trayecto se instalaron marcas visibles cada 3 metros para facilitar el registro de la distancia recorrida. La prueba fue realizada por profesores de educación física entre las 17:00 y las 20:00 horas, y utilizaron frases motivacionales planteadas en el protocolo. En una ficha diseñada especialmente para la prueba se registraron datos personales, peso y talla. Luego de un reposo de 10 minutos, se explicó en forma clara e individual las características de la prueba. Se registraron, antes del 6MWT, al final y a los 3 minutos posteriores, la frecuencia respiratoria, la frecuencia cardiaca y la saturación de oxígeno ${ }^{15}$.

Se aplicaron seis a ocho ejercicios de sobrecarga de los grandes grupos musculares (flexores y extensores del antebrazo, flexores de tronco, pectorales, elevadores del hombro, extensores de rodilla y plantiflexores) y se ejecutaron tres series durante 60 segundos por cada una, con una intensidad que inducía fallo muscular al final de este periodo y con 2 minutos de pausa entre series (metodología denominada
$1 \times 2 \times 3)^{16}$; semanalmente se incorporaron de 2 a $4 \mathrm{~kg}$, alcanzando siempre el tiempo de ejercicio señalado en el método. Cada sesión incluyó 10 minutos de un calentamiento que consistió en carrera continua, ejercicios de locomoción y movilidad articular, desplazamientos laterales y elongación. Al finalizar la fase principal se realizó un enfriamiento y estiramiento tras la sesión.

Todas las variables se expresaron como media y desviación típica. La normalidad de las variables se evaluó a través de la prueba Shapiro Wilk. Para la comparación de las variables antes y después de la intervención y entre grupos se utilizó el test t de Student para muestras relacionadas y para muestras independientes. Todos los análisis se realizaron con el programa SPSS, versión 23,0 . Se utilizó un margen de error del $5 \%(p<0.05)$.

\section{Resultados}

En el grupo adherente, el peso $(p<0.001)$, el IMC $(p<0.001)$ y el CC $(p=0.001)$ presentaron cambios significativos con el programa de ejercicio físico. La capacidad cardiorrespiratoria aumentó significativamente $(9.80 \% ; p=0.001)$. La presión arterial sistólica no presentó cambios significativos ( $p \geq 0.05$ ), y en cambio la presión diastólica $(p=0.011)$ y la glucemia basal disminuyeron significativamente $(p=0.021)$. En el grupo no adherente disminuyeron el peso, el IMC y el CC, alcanzado significancia estadística $(p<0.05)$, pero resultados porcentuales inferiores a los del grupo adherente. En la comparación entre grupo adherente y no adherente solo hubo diferencias significativas en pretest del IMC ( $p$ < 0.05) (Tabla 1).

En la tabla 2 se aprecia que, en el grupo adherente, los triglicéridos disminuyeron significativamente ( $p<0.001$ ) un $20.46 \%$; en cambio, el colesterol total, el c-HDL y el c-LDL no presentaron variaciones significativas $(p \geq 0.05)$. El grupo no adherente no presentó variaciones significativas en el perfil lipídico ( $p \geq 0.05$ ); además, su c-HDL disminuyó un $12,01 \%$. No hubo diferencias significativas en la comparación entre grupos.

\section{Discusión}

El propósito de investigación fue evaluar los efectos de un programa de ejercicio físico sobre el perfil lipídico, el estado ponderal y la salud cardiovascular de obesos candidatos a cirugía bariátrica. Al no existir 
Gaceta Médica de México. 2017;153

Tabla 1. Resultado comparativo del peso, IMC y CC

\begin{tabular}{|c|c|c|c|c|}
\hline & & Grupo adherente & Grupo no adherente & $\begin{array}{c}\mathrm{p} \\
\text { (entre grupos) }\end{array}$ \\
\hline \multirow[t]{4}{*}{ Peso (kg) } & Pretest & $119.05 \pm 23.68$ & $103.05 \pm 9.71$ & 0.128 \\
\hline & Postest & $108.91 \pm 20.28$ & $100.50 \pm 10.15$ & 0.348 \\
\hline & Cambio (\%) & -8.52 & -2.91 & \\
\hline & $p$ & $<0.001$ & 0.006 & \\
\hline \multirow[t]{4}{*}{$\mathrm{IMC}\left(\mathrm{kg} / \mathrm{m}^{2}\right)$} & Pretest & $44.41 \pm 7.71$ & $39.78 \pm 2.16$ & 0.041 \\
\hline & Postest & $40.61 \pm 6.44$ & $38.78 \pm 2.25$ & 0.510 \\
\hline & Cambio (\%) & -8.55 & -2.56 & \\
\hline & $p$ & $<0.001$ & 0.007 & \\
\hline \multirow[t]{4}{*}{$\mathrm{CC}(\mathrm{cm})$} & Pretest & $131.56 \pm 17.24$ & $123.50 \pm 6.57$ & 0.284 \\
\hline & Postest & $123.19 \pm 12.63$ & $120.67 \pm 6.71$ & 0.650 \\
\hline & Cambio (\%) & -6.37 & -2.43 & \\
\hline & $p$ & 0.001 & 0.047 & \\
\hline \multirow[t]{4}{*}{ Presión diastólica (mmHg) } & Pretest & $83.00 \pm 8.61$ & $75.33 \pm 5.72$ & 0.070 \\
\hline & Postest & $75.29 \pm 8.13$ & $76.83 \pm 2.99$ & 0.660 \\
\hline & Cambio (\%) & -9.29 & $1.34 \%$ & \\
\hline & $p$ & 0.011 & 0.472 & \\
\hline \multirow[t]{4}{*}{ Presión sistólica (mmHg) } & Pretest & $126.77 \pm 16.20$ & $120.00 \pm 22.92$ & 0.467 \\
\hline & Postest & $121.75 \pm 8.53$ & $126.40 \pm 8.85$ & 0.327 \\
\hline & Cambio (\%) & -3.96 & $5.03 \%$ & \\
\hline & $\mathrm{p}$ & 0.312 & 0.590 & \\
\hline \multirow[t]{4}{*}{ 6MWT (m) } & Pretest & $568.57 \pm 52.27$ & $577.50 \pm 73.74$ & 0.760 \\
\hline & Postest & $624.29 \pm 77.16$ & $615.83 \pm 100.52$ & 0.839 \\
\hline & Cambio (\%) & 9.80 & 6.60 & \\
\hline & $\mathrm{p}$ & 0.001 & 0.181 & \\
\hline \multirow[t]{4}{*}{ Glucemia basal (mg/dl) } & Pretest & $100.93 \pm 15.19$ & $94.50 \pm 4.14$ & 0.326 \\
\hline & Postest & $95.29 \pm 11.96$ & $93.67 \pm 3.39$ & 0.751 \\
\hline & Cambio (\%) & -5.60 & 1.08 & \\
\hline & $p$ & 0.021 & 0.259 & \\
\hline
\end{tabular}

CC: contorno de la cintura; IMC: índice de masa corporal; 6MWT: test de la marcha de 6 minutos.

Tabla 2. Comparación (media y desviación típica) de triglicéridos, colesterol total, c-HDL y c-LDL

\begin{tabular}{|c|c|c|c|c|}
\hline & & Grupo adherente & Grupo no adherente & $\begin{array}{c}\mathrm{p} \\
\text { (entre grupos) }\end{array}$ \\
\hline \multirow[t]{4}{*}{ Triglicéridos (mg/dl) } & Pretest & $145.44 \pm 59.49$ & $122.37 \pm 25.63$ & 0.711 \\
\hline & Postest & $115.69 \pm 52.35$ & $116.50 \pm 25.84$ & 0.570 \\
\hline & Cambio (\%) & -20.46 & $3.34 \%$ & \\
\hline & $p$ & $<0.001$ & 0.365 & \\
\hline \multirow[t]{4}{*}{ Colesterol total (mg/dl) } & Pretest & $180.87 \pm 43.12$ & $180.33 \pm 20.15$ & 0.851 \\
\hline & Postest & $175.80 \pm 37.53$ & $177.80 \pm 22.30$ & 0.661 \\
\hline & Cambio (\%) & -2.80 & $-1.66 \%$ & \\
\hline & $p$ & 0.602 & 0.364 & \\
\hline \multirow[t]{4}{*}{$\mathrm{c}-\mathrm{HDL}(\mathrm{mg} / \mathrm{dl})$} & Pretest & $43.63 \pm 10.07$ & $50.67 \pm 20.92$ & 0.239 \\
\hline & Postest & $45.00 \pm 9.91$ & $44.00 \pm 7.40$ & 0.825 \\
\hline & Cambio (\%) & 3.15 & $-12.01 \%$ & \\
\hline & $p$ & 0.423 & 0.410 & \\
\hline \multirow[t]{4}{*}{ c-LDL (mg/dl) } & Pretest & $113.31 \pm 41.60$ & $134.33 \pm 67.75$ & 0.626 \\
\hline & Postest & $109.59 \pm 38.85$ & $131.33 \pm 67.93$ & 0.088 \\
\hline & Cambio (\%) & -3.29 & -2.23 & \\
\hline & $\mathrm{p}$ & 0.602 & 0.229 & \\
\hline
\end{tabular}


grupo de control, se compararon dos grupos según participación en el programa; aquellos que no cumplieron con los porcentajes de asistencia fueron agrupados como no adherentes. Los hallazgos más importantes fueron encontrados en el grupo adherente, en el que se observó una reducción significativa del peso $(p<0.001)$, el IMC $(p<0.001)$, el CC $(p=0.001)$, la presión diastólica $(p=0.011)$ y los triglicéridos $(p$ $<0.001$ ), y un aumento significativo de los metros recorridos en el 6TMW ( $p=0.001)$. El grupo no adherente presentó solo cambios significativos en peso, IMC y CC, pero porcentualmente muy inferiores a los del grupo adherente.

Los participantes fueron concientizados de no participar en ningún otro protocolo de ejercicio que estuviera estructurado y se les recomendó mantener sus patrones normales durante la intervención. Con el programa, el peso disminuyó de forma significativa, aplicando en los pacientes ejercicios intervalados. Una investigación realizada por Sánchez, et al. ${ }^{17}$ reportó disminuciones de $5.17 \mathrm{~kg}$ en promedio en pacientes obesos mórbidos pendientes de cirugía aplicando un programa de 2 meses de duración con ejercicio de intensidad moderada. Un estudio realizado durante 3 meses en obesos mórbidos y con comorbilidad, en los que se aplicó ejercicio intervalado con metodología similar a la utilizada en la presente investigación, obtuvo una disminución de $1.41 \mathrm{~kg}^{18}$. Al comparar los resultados de este estudio con una investigación de igual tiempo de duración donde aplicaban ejercicio físico aeróbico, tuvieron una disminución de $5.3 \mathrm{~kg}^{19}$, valores inferiores a los $10.14 \mathrm{~kg}$ reportados en esta investigación. La disminución del peso antes de la cirugía bariátrica se asocia con una reducción notable del riesgo de complicaciones posoperatorias ${ }^{20}$ y con mejoras en la morfología y en la función cardiaca, así como con una reducción de los factores de riesgo cardiovascular ${ }^{21}$.

Un exceso de masa grasa abdominal y visceral se considera, en los estudios epidemiológicos, como uno de los más importantes factores de riesgo de enfermedad cardiovascular ${ }^{22,23}$, por lo que la disminución del CC debe ser un objetivo importante en los programas de intervención en este tipo de pacientes. En el presente estudio, el CC disminuyó $8.37 \mathrm{~cm}$ en el grupo adherente, mejorando las condiciones y la salud de los participantes; resultados similares a los reportados en una investigación realizada en mujeres candidatas a cirugía bariátrica $(7.57 \mathrm{~cm})^{24}$ y a los de una intervención en el estilo de vida de obesos mórbidos que implicó dieta combinada con actividad física y que obtuvo cambios favorables en los factores de riesgo cardiometabólico ${ }^{25}$. Por lo tanto, la actividad física promueve una mayor reducción en el CC y en el contenido de grasa hepática.

La presión diastólica se redujo significativamente, pero la presión sistólica no alcanzó significancia, aunque disminuyó un $3.96 \%$, en comparación con un programa de ejercicio aeróbico de 6 meses de duración que presentó cambios significativos en la presión sistólica $(-23.8 \pm 27.7 \mathrm{mmHg} ; \mathrm{p}<0.0001)$ y diastólica $(-14.4 \pm 8.9 \mathrm{mmHg} ; \mathrm{p}<0.0001)^{19}$. Intervenciones con asesorías nutricionales y cambios en la actividad física de 1 año de duración han presentado resultados positivos en estas variables de estudio ${ }^{26}$, mejorando la salud cardiovascular de los participantes con malnutrición por exceso.

La capacidad cardiorrespiratoria aumentó significativamente en un $9.80 \%$; importante variación, ya que esta se asocia con un menor riesgo cardiometabóli$\mathrm{co}^{27}$. En estudios de 4 meses de duración con abordaje multidisciplinario, la distancia recorrida en el 6MWT se incrementó un $7.17 \%$. Por otro lado, un protocolo de intervención de 8 semanas de ejercicio intervalado de alta intensidad y de ejercicio físico de sobrecarga mejoró significativamente el rendimiento físico aeróbico de mujeres con y sin alteraciones metabólicas ${ }^{29}$.

En el perfil metabólico, la glucemia basal disminuyó un $5.60 \%$, similar a otras investigaciones realizadas en mujeres sedentarias con sobrepeso y obesidad a las que se aplicaban ejercicios de alta intensidad ${ }^{30}$, pero inferior a lo reportado en otros grupos de obesos mórbidos $^{24,31}$. En el perfil lipídico, los triglicéridos disminuyeron significativamente un $20.46 \%$, y el colesterol total, el c-HDL y el c-LDL no presentaron variaciones significativas; resultados similares a los descritos en otras investigaciones ${ }^{24}$. Estudios con una duración de 6 y 12 meses con control de la dieta y ejercicio físico provocaron disminuciones de los parámetros metabólicos en sujetos con obesidad y obesidad mórbida ${ }^{25,32}$; por lo tanto, las intervenciones sobre el estilo de vida, con ejercicio físico estructurado, se pueden considerar como el pilar del tratamiento de la obesidad mórbida.

En conclusión, el programa presenta beneficios para el tratamiento de esta condición, sin efectos adversos para la salud de los participantes, y es factible de realizar debido a los bajos costos que conlleva. Se pretende, para futuras investigaciones, incrementar el número de participantes y comparar métodos con distintas intensidades de trabajo. 


\section{Bibliografía}

1. Myers M, Leibel R, Seeley R, et al. Obesity and leptin resistance: distinguishing cause from effect. Trends Endocrinol Metab 2010;21: 643-51.

2. Morton G, Schwartz M. Leptin and the central nervous system control of glucose metabolism. Physiol Rev. 2011;91:389-411.

3. Kushner RF. Weight loss strategies for treatment of obesity. Prog Cardiovasc Dis. 2014;56:465-72.

4. Melanson KJ, Mclnnis KJ, Rippe JM, et al. Obesity and cardiovascula disease risk: research update. Cardiol Rev. 2001;9:202-7.

5. Dallongeville J, Bringer J, Bruckert E, et al. Abdominal obesity is associated with ineffective control of cardiovascular risk factors in primary care in France. Diabetes Metab. 2008;34:606-11.

6. Calle EE, Thun MJ. Obesity and cancer. Oncogene. 2004;23:6365-78.

7. Muñoz M, Mazure R, Culebras J. Obesidad y sistema inmune. Nutr Hosp. 2004;19:319-24.

8. Karamouzis I, Pervanidou P, Berardelli $R$, et al. Enhanced oxidative stress and platelet activation combined with reduced antioxidant capacity in obese prepubertal and adolescent girls with full or partial metabolic syndrome. Horm Metab Res. 2011;43:607-13.

9. Fontaine KR, Redden DT, Wang C, et al. Years of life lost due to obesity. JAMA. 2003;289:187-93.

10. Ruano M, Silvestre V, Aguirregoicoa E, et al. Nutrición, síndrome metabólico y obesidad mórbida. Nutr Hosp. 2011;26:759-64.

11. MINSAL. Encuesta Nacional de Salud 2009-2010. Santiago: Ministerio de Salud de Chile; 2010.

12. Ocón J, Pérez S., Gimeno S, et al. Eficacia y complicaciones de la cirugía bariátrica en el tratamiento de la obesidad mórbida. Nutr Hosp. 2005;20:409-14.

13. Lanzarini E, Marambio A, Fernández L, et al. Hiperobesidad y obesidad mórbida: estudio comparativo. Rev Chil Cir. 2012;64:233-7.

14. MINSAL. Guía clínica de hipertensión arterial primaria o esencial en personas de 15 años y más. Santiago: Ministerio de Salud de Chile; 2010.

15. Gatica D, Puppo H, Villarroel G, et al. Valores de referencia del test de marcha de seis minutos en niños sanos. Rev Méd Chile. 2012;140:1014-21.

16. Saavedra C. Guía de actividad física para el adulto mayor. Santiago: Instituto Nacional del Deporte; 2006.

17. Sánchez L, Sánchez C, García A. Valoración de un programa de ejercicio físico estructurado en pacientes con obesidad mórbida pendientes de cirugía bariátrica. Nutr Hosp. 2014;29:64-72.

18. Delgado $P$, Jerez $D$, Caamaño $F$, et al. Doce semanas de ejercicio físico intervalado con sobrecarga mejora las variables antropométricas de obesos mórbidos y obesos con comorbilidades postulantes a cirugía bariátrica. Nutr Hosp. 2015;32:2007-11.
19. Marcon E, Gus I, Neumann C. Impacto de um programa mínimo de exercícios físicos supervisionados no risco cardiometabólico de pacientes com obesidade mórbida. Arq Bras Endocrinol Metab. 2011;55:331-8.

20. Anderin C, Gustafsson UO, Heijbel N, et al. Weight loss before bariatric surgery and postoperative complications: data from the Scandinavian Obesity Registry (SOReg). Ann Surg. 2015;261:909-13.

21. Rueda-Clausen CF, Ogunleye AA, Sharma AM. Health benefits of longterm weight-loss maintenance. Annu Rev Nutr. 2015;35:475-516.

22. Despres JP, Lemieux I, Bergeron J, et al. Abdominal obesity and the metabolic syndrome: contribution to global cardiometabolic risk. Arterioscler Thromb Vasc Biol. 2008;28:1039-49.

23. Sung RY, Yu CC, Choi KC, et al. Waist circumference and body mass index in Chinese children: cutoff values for predicting cardiovascular risk factors. Int J Obes (Lond). 2007;31:550-8.

24. Delgado $P$, Jerez D, Caamaño $F$, et al. Efectividad del tratamiento integral sobre las condiciones preoperatorias de mujeres obesas candidatas a cirugía bariátrica. Nutr Hosp. 2015;32:2570-5.

25. Goodpaster BH, Delany JP, Otto AD, et al. Effects of diet and physical activity interventions on weight loss and cardiometabolic risk factors in severely obese adults: a randomized trial. JAMA. 2010;304: $1795-802$.

26. Unick J, Beavers D, Jakicic J, et al. Effectiveness of lifestyle interventions for individuals with severe obesity and type 2 diabetes results from the look AHEAD trial. Diabetes Care. 2011;34:2152-7.

27. Díez-Fernández A, Sánchez-López M, Mora-Rodríguez R, et al. Obesity as a mediator of the influence of cardiorespiratory fitness on cardiometabolic risk: a mediation analysis. Diabetes Care. 2014;37:855-62.

28. Delgado $\mathrm{P}$, Cofré $\mathrm{A}$, Alarcón $\mathrm{M}$, et al. Evaluación de un programa integral de cuatro meses de duración sobre las condiciones preoperatorias de pacientes obesos candidatos a cirugía bariátrica. Nutr Hosp. 2015;32:1022-7.

29. Álvarez C, Ramírez-Campillo R, Henríquez-Olguín C, et al. ¿Pueden ocho semanas de ejercicio físico combinado normalizar marcadores metabólicos de sujetos hiperglicémicos y dislipidémicos? Rev Méd Chile. $2014 ; 142: 458-66$

30. Álvarez C, Ramírez $\mathrm{R}$, Flores $\mathrm{M}$, et al. Efectos del ejercicio físico de alta intensidad y sobrecarga en parámetros de salud metabólica en mujeres sedentarias, pre-diabéticas con sobrepeso u obesidad. Rev Méd Chile. 2012:140:1289-96.

31. Delgado $P$, Caamaño $F$, Jerez $D$, et al. Efectos de un programa de tratamiento multidisciplinar en obesos mórbidos y obesos con comorbilidades candidatos a cirugía bariátrica. Nutr Hosp. 2015;31:2014-9.

32. Miller CT, Fraser SF, Straznicky NE, et al. Effect of diet versus diet and exercise on weight loss and body composition in class ii and iii obesity: a systematic review. J Diabetes Metab. 2013;4:1-6. 